\title{
Investigation of the relationships between acoustic attenuation and ionic conduction of metaphosphate glasses
}

\author{
Laura Muñoz-Senovilla ${ }^{1}$, Jana Bírešová $^{2}$, Peter Hockicko ${ }^{2}$, Francisco Muñoz ${ }^{1 *}$ \\ ${ }^{1}$ Ceramics and Glass Institute (CSIC), Kelsen 5, 28049 Madrid, Spain \\ ${ }^{2}$ Department of Physics, Faculty of Electrical Engineering, University of Žilina, Zilina, \\ Slovakia
}

\begin{abstract}
This paper deals with phosphate-based glasses and summarizes the results obtained from acoustic attenuation and ionic conduction investigations. Several relaxation processes have been found to be connected with different activation energies, for which Double Power Law and Gaussian functions have been used for their modelling. The activation energy of the acoustic relaxations in the metaphosphate glasses increases with the increasing cationic potential of the modifier elements, following a direct relationship between the cationic potential of the modifier in the glasses and their influence on the short and medium range structural arrangements of the $\mathrm{PO}_{4}$ tetrahedra. On the other hand, the variation of the activation energy for the ionic conduction shows a similar trend than those observed in the acoustic attenuation processes, which indicates that both phenomena are closely influenced by the short and medium range structure of the glasses, particularly through the strength of the modifier-oxygen bonds and the glass network reticulation.
\end{abstract}


Keywords: Phosphate Glasses; Conductivity; Acoustic Attenuation; Structure; Relaxation

*Corresponding author's e-mail: $\underline{\text { fmunoz@icv.csic.es }}$ 


\section{Introduction}

The study of the fundamental properties and relaxation phenomena of phosphate based glasses has gained very much interest in the recent years due to their special chemical and physical characteristics. Even though they often suffer of a limited chemical resistance because of their high hygroscopicity, phosphate glasses have found application fields where they are more suitable than their silicate counterparts. For instance, they are used in very diverse applications such as biomedicine [1], for the storage of radioactive wastes [2] or for the application as high power lasers host materials [3].

The network of phosphate glasses is known to be built up of $\mathrm{PO}_{4}$ tetrahedra, which can be represented by the $\mathrm{Q}^{\mathrm{n}}$-type groups, according to the nomenclature of Lippmaa et al. [4], where $\mathrm{n}$ is the number of bridging oxygen atoms. Thus, metaphosphate glasses, where the $\mathrm{O} / \mathrm{P}$ ratio equals 3 , have a structure based on chains or rings of $\mathrm{Q}^{2}$-type species, i.e. having two bridging (BO) and two non-bridging (NBO) oxygens [5]. These chains and rings are connected through non-bridging oxygen atoms to the modifier cations.

Recently, Nuclear Magnetic Resonance (NMR) and Raman spectroscopy were used to get more insights into the relationships between the structure of alkali and alkaline-earth and zinc metaphosphate glasses and their viscosity behaviour [6]. It has been found that there is a close relationship between the strength of the metal-oxygen bonds and the activation energy for the viscous flow. The activation energy seems to be much influenced not only by the bond strength but also through the molar volume of the glasses, suggesting that the medium range order structure of the phosphate network, as 
represented in the form of chains or rings, does also play a key role in the rheological behaviour within the temperature range studied.

As it has been demonstrated previously, acoustic waves are suitable not only for the investigation of materials directly related to acoustics, but also to study the properties of semiconductor structures [7] and glasses [8-12]. In the particular case of lithium and copper bearing phosphate glasses, the authors showed previously that there seems to be a close correlation between the activation energy for the ionic conduction and the activation energy of the acoustic relaxation process below the glass transition temperature [8-9]. In these glasses, the temperature dependences of acoustic attenuation generally showed one or more peaks which have been attributed to different relaxation mechanisms related to ion hopping processes of mobile cations, the glass network structure and dynamics connected with local distorsions and diffusion [13]. As Bridge et al. observed [10], the absorption of the acoustic waves as a function of temperature has been ascribed to the presence of particles or groups of particles moving in double-well potentials.

The aim of the present work was to investigate the possible relationships existing between both acoustic attenuation and ionic conduction properties and how the short and medium range structure of the previously studied metaphosphate glasses may have an influence on them.

\section{Experimental}

Alkali, alkaline-earth and zinc metaphosphate glasses were prepared by conventional melt-quenching technique as previously described in [6]. Stoichiometric amounts of reagent grade carbonates and $\left(\mathrm{NH}_{4}\right)_{2} \mathrm{HPO}_{4}$ (Merck, 99\%) were weighed and mixed. The 
batches were calcined in porcelain crucibles, held in an electric furnace up to $450^{\circ} \mathrm{C}$, and then melted in an electric furnace during $2 \mathrm{~h}$ at temperatures ranging from $800^{\circ} \mathrm{C}$ to $1100^{\circ} \mathrm{C}$ depending on the composition. The melts were poured onto brass moulds and annealed slightly above their glass transition temperatures $\left(\mathrm{T}_{\mathrm{g}}\right)$. The glass transition temperature was determined using the thermal expansion curves of the glasses obtained in air with a Netzsch Gerätebau dilatometer, model $402 P C / 1$ at a heating rate of 2 K.min ${ }^{-1}$. Prismatic samples around $10 \mathrm{~mm}$ in length were used for the measurements. Longitudinal acoustic waves of $13 \mathrm{MHz}$ frequency generated by the Modulator and receiver MATEC 7700 and $\mathrm{LiNbO}_{3}$ transducer acoustically coupled directly to the sample were used to investigate the glasses. Acoustic measurements were performed at temperatures ranging from $290 \mathrm{~K}$ to $650 \mathrm{~K}$ at a heating rate of $0.5 \mathrm{~K} \cdot \mathrm{min}^{-1}$. A computer with system LabVIEW 2010 was employed and the data were processed from digital multimeters. An HP digital multimeter measures the temperature of the sample using a thermocouple type $\mathrm{T}$, and the measured voltage is converted to temperature using Newton interpolation method. A second multimeter measures the output voltage from Box-Car which corresponds to the attenuation of acoustic waves. The prepared samples were either cylindrical or square in shape (thickness $\mathrm{h}=2.83$ to $3.54 \mathrm{~mm}$ and 8.9 to 9.1 $\mathrm{mm}$ in diameter for the cylindrical samples and area $\mathrm{S}=7.1 \times 7.6 \mathrm{~mm}^{2}$ for square samples). Both faces were cut plane-parallel and polished.

The electrical conductivity of the glasses was calculated through the determination of the bulk resistance by Electrochemical Impedance Spectroscopy in a VMP3 potentiostat from BioLogic, within the frequency range of $10 \mathrm{~Hz}$ to $1 \mathrm{MHz}$. For the measurements, gold electrodes were deposited onto both faces of ca. $1 \mathrm{~cm}^{2}$ square and 2 to $3 \mathrm{~mm}$ thick samples. The resistance of the sample is read in the Nyquist plots of the complex versus 
real impedance at the intersection point with the horizontal axis and the conductivity is calculated using the equation:

$$
\sigma=(1 / \mathrm{R})(\mathrm{L} / \mathrm{A})\left({\mathrm{S} . \mathrm{cm}^{-1}}^{-1}\right.
$$

being L/A the sample geometric factor of the sample, where $\mathrm{L}$ is thickness and $\mathrm{A}$ is sample area.

\section{Results}

Figure 1 shows the measured acoustic spectra of the alkaline-earth and zinc bearing investigated glasses. The relaxation processes characterized by the activation energy $\mathrm{E}_{\mathrm{a}}$ for jumps over the barrier between two potential minima as well as the typical relaxation frequency of ion hopping, $v_{0}=1 / \tau_{0} \approx 10^{13}-10^{14} \mathrm{~s}^{-1}$, can be described by the Arrhenius relation between peak temperature $\mathrm{T}_{\text {peak }}$ and applied acoustic frequency $v$ $[14,15]$. From the temperature dependences of acoustic attenuation and positions of the peaks, the activation energy values of dominant processes can be determined by using equation (2):

$$
v=v_{0} \exp \left(-\frac{E_{a}}{k_{B} T_{\text {peak }}}\right)
$$

where $v$ is the characteristic relaxation frequency, $v_{0}$ is the pre-exponential factor, $\mathrm{E}_{\mathrm{a}}$ is the activation energy of the relaxation process, $k_{B}$ is the Boltzmann constant and $T_{\text {peak }}$ is the temperature of peak maxima. Theoretical analysis using Double Power Law (DPL) function (Eq. 3) has given an excellent fit of acoustic attenuation of the different kinds of sites which are responsible for the ionic hopping motion (Fig. 2a), as was pointed in [19]. 
$\alpha(\omega, T) \propto \sum_{i=1}^{n} \frac{1}{(\omega \tau)^{-p}+(\omega \tau)^{q}}$

where $n$ is the number of peaks to fit and $p, q$ are power exponents.

The acoustic attenuation will exhibit a maximum when $\omega \tau=1$, where $\omega$ is the angular frequency and $\tau$ is relaxation time [19].

Comparatively speaking, the sample $50 \mathrm{CaO} .50 \mathrm{P}_{2} \mathrm{O}_{5}$ can be fit similarly by four overlapping Gaussian functions (Fig. 2b), as described by equation (4),

$\alpha=\sum_{i=1}^{n} a_{i} e^{\left[-\left(\frac{x-b_{i}}{c_{i}}\right)^{2}\right]}$

where $a$ is amplitude, $b$ is centroid (location), $c$ is related to the peak width, $n$ is the number of peaks to fit and $x$ is an independent parameter of temperature dependence of measured acoustic attenuation.

Absorption peaks can be modelled by means of DPL or Gaussian functions and so the activation energy of the different relaxation processes calculated. Figure $2(a, b)$ shows the modelling using DPL and Gauss functions for the glass with composition $\mathrm{Ca}\left(\mathrm{PO}_{3}\right)_{2}$. As it can been seen, using different functions, the very close values of activation energies of the same processes were estimated. Both models give a good fit of experimental data and their estimations of activation energies are really close.

Figure 3 presents the activation energy values obtained for both the high $\left(E_{a} \_1\right)$ and low temperature $\left(E_{a} 2\right)$ peaks in the acoustic attenuation spectra of the glasses against the modifier cationic potential. The data for $\mathrm{LiPO}_{3}$ glass has been taken from a previous work [16]. The dependence of $E_{a}$ of the highest temperature peak (A1) with $Z / a$ has 
increasing tendency though it decreases slightly from $\mathrm{Li}$ to $\mathrm{Ba}$ glasses. This discontinuity has been seen also in the kinetic fragility of these glasses [6]. However, the $\mathrm{E}_{\mathrm{a}}$ of the low temperature peak (A2) presents a sharper decrease from Li to $\mathrm{Ba}$ and a more irregular increase from $\mathrm{Ba}$ to $\mathrm{Zn}$, decreasing again from $\mathrm{Zn}$ to $\mathrm{Mg}$.

Figure 4 gathers the Arrhenius plots for conductivity of the studied glasses, lithium, sodium, alkaline-earth and zinc metaphosphates. It can be clearly seen that the alkali containing glasses have the highest values of conductivity among all the compositions and that the conductivity of the $\mathrm{Zn}\left(\mathrm{PO}_{3}\right)_{2}$ is higher than that of the alkaline-earth phosphate glasses.

In Figure 5 the activation energy for the ionic conduction has been plotted against the cationic potential of the modifier ion in each composition. At first sight, there seems to be a correlation between the activation energy for ionic conduction and the cationic potential, i.e. the higher the cationic potential the higher the $\mathrm{E}_{\mathrm{a}}$, with two exceptions for the glasses $\mathrm{LiPO}_{3}$ and $\mathrm{Zn}\left(\mathrm{PO}_{3}\right)_{2}$. The activation energy decreases from sodium to lithium due to the much smaller size of the cation, even though Li has higher bond strength with the surrounding oxygen atoms. Zinc metaphosphate glass posseses unusual lower glass transition temperature than the one that would be expected according to the cationic potential of $\mathrm{Zn}$ and, as in the present case, a lower activation energy for ionic conduction. This is supposed to be due to the higher stability of the $\mathrm{d}^{10}$ electronic configuration of the $\mathrm{Zn}^{2+}$ ions, which may give rise to a lower strength of the $\mathrm{Zn}-\mathrm{O}$ bonds when compared to the bivalent alkaline-earth modifiers. From $\mathrm{Ba}$ to $\mathrm{Mg}$ alkaline-earth series, the activation energy for conduction increases with the cationic 
potential as it is expected from the point of view of the increased metal-oxygen bond strength in the order $\mathrm{Ba}<\mathrm{Sr}<\mathrm{Ca}<\mathrm{Mg}$.

\section{Discussion}

Ionic conducting glasses investigated so far by acoustic attenuation show broad peaks and located below the glass transition temperature of the glass at the characteristic temperature of the maximum of the peak $\left(T_{\text {peak }}\right)$. Their occurrence has been thought to be connected to the activation energy of the ionic hopping process of the modifier cations involved in the conduction mechanism [19]. For the alkaline-earth and zinc bearing glasses particularly, this temperature of peak maximum increases in the order $\mathrm{Ba}<\mathrm{Sr}<\mathrm{Ca}<\mathrm{Zn}<\mathrm{Mg}$ (Fig. 1). As it is also known, the cationic potential of the modifier ions ( $Z / a)$, where $Z$ is the charge and $a$ is the ionic radius, increases in the same sequence and the glass transition of such glass compositions showed a similar variation as published before in [6]. Therefore, in a first stage it can be said that the relaxation processes taking place through the acoustic attenuation measurements are related to the bonding strength of the glass network as the glass transition does.

Muñoz-Senovilla et al. showed that, generally, the kinetic fragility of the glasses, which measures the variation rate of viscosity with temperature at the glass transition temperature [17], increases with the cationic potential as well, though this takes higher values for the alkali containing glasses than for the alkaline-earth ones. The authors proposed that there is a quite important influence of the relationship between the bond strength of the glass network and the molar volume of the glasses, being this a determinant factor that may explain the change in the tendency observed between the $\mathrm{Li}$ and $\mathrm{Ba}$ metaphosphate glasses. From Na to Li metaphosphate compositions, the kinetic fragility increases, as it also does from $\mathrm{Ba}$ to $\mathrm{Mg}$ series. However, there is a sudden 
decrease when going from $\mathrm{LiPO}_{3}$ to $\mathrm{Ba}\left(\mathrm{PO}_{3}\right)_{2}$ glasses. This discontinuity in the tendency has been explained through the much higher molar volume of the barium metaphosphate glass [6]. On the other hand, Zn metaphosphate glass shows always slightly different values with respect to the variation in the $\mathrm{M}^{2+}$ metaphosphate series, which is explained as due to the different stability of the $\mathrm{Zn}-\mathrm{O}$ bonds due to the particular electronic configuration of $\mathrm{Zn}^{2+}$ ions. Furthermore, the $\mathrm{Zn}\left(\mathrm{PO}_{3}\right)_{2}$ glass possesses a similar average single bond strength than that of the alkaline-earth ones but a much higher molar volume, thus giving rise to a different behaviour in their transport properties, such as the viscosity or the ionc conduction as it is shown below.

The variation of the activation energy for the ionic conduction process can easily be explained using the model of Anderson \& Stuart [18], which uses a decomposition of the total activation energy for the conduction in two parts, one arising from the bond strength of the ion to the surrounding oxygens, and a second one influenced by the glass network strength determining the easiness for the diffusion of the charge carriers. Thus, the higher the cationic potential the higher the metal-oxygen bond strength is, and so this will contribute to the increase of the total activation energy. However, other factors like the much smaller size of the lithium ions and the polarizability of the zinc have a great influence into the conduction mechanism and are then responsible of their lower values.

In the same work [6], Muñoz-Senovilla et al. published the Raman spectra of the series of metaphosphate glasses and showed the variation of the Raman shifts attributed to the symmetric stretching vibrations of the O-P-O bonds in the form of either chains or rings. They both increase with the cationic potential of the modifier, except between Li and $\mathrm{Ba}$ glasses, where there is a slight decrease. This decrease is also seen in the variation of the activation energy of the high temperature peak. Therefore, it is thought 
that there must also be a close relationship between the average single bond strength of the glass network, as represented by the Raman shift variation with composition, and the activation energy of acoustic attenuation.

Another interesting observation from the attenuation plots is the ratio between the areas under the relaxation peaks that have been obtained below the glass transition temperature. Figure 6 shows the ratio between the areas of the low temperature peak (A2) and the high temperature peak (A1) found in acoustic attenuation (Fig. 2) against the cationic potential. The data point for the $\mathrm{NaPO}_{3}$ composition has been omitted because it was only possible to determine the A1 peak. It can be seen that there is first a decrease of $\mathrm{A} 2 / \mathrm{A} 1$ ratio between $\mathrm{LiPO}_{3}$ and $\mathrm{Zn}\left(\mathrm{PO}_{3}\right)_{2}$, then a sharp increase from $\mathrm{Zn}$ to $\mathrm{Mg}$ bearing glasses. This relationship could be understood in terms of a different contribution from each relaxation process if those were attributed to the hopping mechanism of ions in different structural rearrangements. However, a more profound analysis of the short and, particularly, medium range order structure of the glasses would be needed in order to get a more precise interpretation.

If one now again looks at the structural data showed in the work of reference [6], particularly the relationship between the Raman shifts of the O-P-O bonds in either chains or rings, it can be seen that there is a decrease from $\mathrm{Na}$ to $\mathrm{Ca}$ metaphosphate glasses, then increasing again to $\mathrm{Zn}$ and $\mathrm{Mg}$, with the cationic potential. This result was interpreted in terms of a variation of the relative proportions of rings and chains in the glasses as a function of the modifier cation, i.e. the proportion of chains increases versus that of the rings with the modifier cationic potential. It is noticeable that the ratio between the areas of the two attenuation peaks varies in the same way that the ratio between the Raman intensities of rings and chains, as pointed out in by MuñozSenovilla et al. [6], i.e. the higher the cationic potential of the modifier the higher the 
proportion of chains in the structure. Therefore, it is thought that the two attenuation peaks observed could correspond to the relaxation of ions involved in $\mathrm{PO}_{4}$ tetrahedra forming either chains, corresponding to the high temperature peak A1, or rings, represented by the low temperature peak A2.

\section{Conclusions}

The acoustic attenuation measurements, studied as a function of temperature, of a series of metaphosphate glasses have shown a connection between the activation energy of the acoustic relaxation processes and that of the ionic conduction through the glass composition and network structure. It was confirmed that the activation energy of the relaxation generally increases with the cationic potential of the modifier element in the composition, and it is associated primarily with an increase of the covalent character of the metal-oxygen bonds, i.e. an higher glass network strength gives rise to an higher activation energy for the acoustic relaxation process. Furthermore, a close similariy has been found between the distribution of chains versus rings at the medium size range order of the structure, as previously studied in the same glasses, and the relative proportions of the relaxation processes occurring in the O-P-O bonds of the chains and rings within the glass network. Meanwhile there can be other specific factors determining directly the activation energy values of the ionic conduction in the glasses, such as in the case of the $\mathrm{Li}$ and $\mathrm{Zn}$ metaphosphates, it cannot be excluded a close relationship between the activation energy of the acoustic relaxation and that of the hopping mechanisms of the ions involved in different structural arrangements of the glass network. 


\section{Acknowledgements}

The authors would like to thank to Mr. F. Černobila for technical assistance. This study was supported by projects KEGA No. 003TU Z-4/2015 and ITMS: 26210120021 cofunded from EU sources and European Regional Development Fund. F. Muñoz and L. Muñoz-Senovilla are thankful to the projects MAT2010-20459 and MAT2013-48246C2-1-P from MINECO of Spain and L. Muñoz-Senovilla also thanks the MINECO for her PhD scholarship (BES-2011-044130). 


\section{References}

[1] I. Ahmed, M. Lewis, I. Olsen, J.C. Knowles, Biomaterials 25 (2004) 491-499.

[2] M.I. Ojovan, W.E. Lee, Metallugircal and Materials Transactions A 42 (2011) 837851.

[3] J.H. Campbell, J.S. Hayden and A. Marker, Int. J. App. Glass Sci. 2[1] (2011) 3-29.

[4] E. Lippmaa, M. Maegi, A. Samoson, G. Engelhardt, A. Grimmer, J. Am. Chem. Soc. 102 (1980) 4889-4893.

[5] R.K. Brow, J. Non-Cryst. Solids 263-264 (2000) 1-28.

[6] L. Muñoz-Senovilla, F. Muñoz, J. Non-Cryst. Solids 385 (2014) 9-16.

[7] P. Hockicko, P. Bury, P. Sidor, H. Kobayashi, M. Takahashi, T. Yanase, Central European Journal of Physics 9 (2011) 242-249.

[8] P. Hockicko, P. Bury, F. Muñoz, J. Non-Cryst. Solids 363 (2013) 140-146.

[9] P. Bury, P. Hockicko, S. Jurecka, M. Jamnicky, Phys. Stat. Sol. 1[11] (2004) 2888 2891.

[10] B. Bridge, A.A. Higazy, J. Mat. Sci. 23 (1988) 438-450.

[11] B. Bridge, N.D. Pater, J. Mat. Sci. 21 (1986) 3783-3800.

[12] R. El-Mallawany, M. Sidkeyb, A. Khafagy and H. Afifib, Mat. Chem. Phys. 37 (1994) 197-200.

[13] M. Cutroni, A. Mandanici, E. Bruno, Phys. Chem. Chem. Phys. 4 (2002) 4539 
[14] B. Roling, A. Happe, M.D. Ingram, K. Funke, J. Phys. Chem. B 103 (1999) 4122 4127.

[15] B. Roling, M.D. Ingram, Phys. Rev. B 57 (1998) 14192-14199.

[16] P. Hockicko, J. Kudelcik, F. Muñoz, L. Muñoz-Senovilla, Advances in Electrical and Electronic Engineering 13[2] (2015) 198-205.

[17] C. A. Angell, Science 267 (1995) 1924-1935.

[18] O.L. Anderson, D.A. Stuart, J. Ame. Ceram. Soc. 37 [12] (1954) 573.

[19] D.P. Almond, A.R. West, Solid State Ionics 26 (1988) 265-278. 


\section{Figure captions}

Figure 1: Acoustic attenuation plots of the alkaline-earth and zinc metaphosphate glasses measured at the frequency of $13 \mathrm{MHz}$.

Figure 2: Temperature dependence of the acoustic attenuation of the glass sample with composition $\mathrm{Ca}\left(\mathrm{PO}_{3}\right)_{2}$ (full line) measured at $13 \mathrm{MHz}$ with the DPL (a) and Gauss (b) fits of the several supposed relaxation processes (dashed line).

Figure 3: Activation energy for the acoustic relaxation peaks at high $\left(\mathrm{E}_{\mathrm{a} \_} 1\right)$ and low $\left(E_{a} \_2\right)$ temperatures against the modifier cationic potential. Lines have been drawn as a guide for the eyes.

Figure 4: Arrhenius plots for conductivity of the alkali and bivalent metaphosphate glasses.

Figure 5: Activation energy values for the ionic conduction as a function of the cationic potential of the modifier atoms. Line has been drawn as a guide for the eyes.

Figure 6: Ratio between the areas of the low (A2) and high temperature (A1) peaks as a function of the cationic potential of the modifier. Line has been drawn as a guide for the eyes.

\section{Table captions}

Table 1. Cationic potential, activation energies of the two dominant relaxation processes connected with the acoustic attenuation peaks A1 and A2, activation energy for the ionic conduction and calculated Log of conductivity at $25^{\circ} \mathrm{C}$ for all metaphosphate glasses. 
Table 1

\begin{tabular}{|l|c|c|c|c|c|}
\hline \multicolumn{1}{|c|}{ Glass } & $\mathrm{Z} / \mathrm{a}\left(\mathrm{A}^{-1}\right)$ & $\begin{array}{c}\mathrm{E}_{\mathrm{a}} \text { attenuation } \\
\text { peak 1 } \\
\pm 0.01 \mathrm{eV})\end{array}$ & $\begin{array}{c}\mathrm{E}_{\mathrm{a}} \text { attenuation } \\
\text { peak 2 } \\
( \pm 0.01 \mathrm{eV})\end{array}$ & $\begin{array}{c}\mathrm{E}_{\mathrm{a}} \text { conduction } \\
( \pm 0.01 \mathrm{eV})\end{array}$ & $\begin{array}{c}\text { Log } \sigma(\sigma \text { in S.cm } \\
@\end{array}$ \\
\hline $\mathrm{NaPO}_{3}$ & 1.02 & 0.69 & n.d. & 0.77 & -7.30 \\
\hline $\mathrm{LiPO}_{3}$ & 1.28 & 0.73 & 0.67 & 0.74 & -6.88 \\
\hline $\mathrm{Ba}\left(\mathrm{PO}_{3}\right)_{2}$ & 1.40 & 0.71 & 0.57 & 1.13 & -16.00 \\
\hline $\mathrm{Sr}\left(\mathrm{PO}_{3}\right)_{2}$ & 1.57 & 0.73 & 0.59 & 1.19 & -16.40 \\
\hline $\mathrm{Ca}\left(\mathrm{PO}_{3}\right)_{2}$ & 1.89 & 0.75 & 0.66 & 1.22 & -17.09 \\
\hline $\mathrm{Zn}\left(\mathrm{PO}_{3}\right)_{2}$ & 2.41 & 0.79 & 0.68 & 1.19 & -16.17 \\
\hline $\mathrm{Mg}\left(\mathrm{PO}_{3}\right)_{2}$ & 2.56 & 0.84 & 0.67 & 1.42 & -20.05 \\
\hline
\end{tabular}




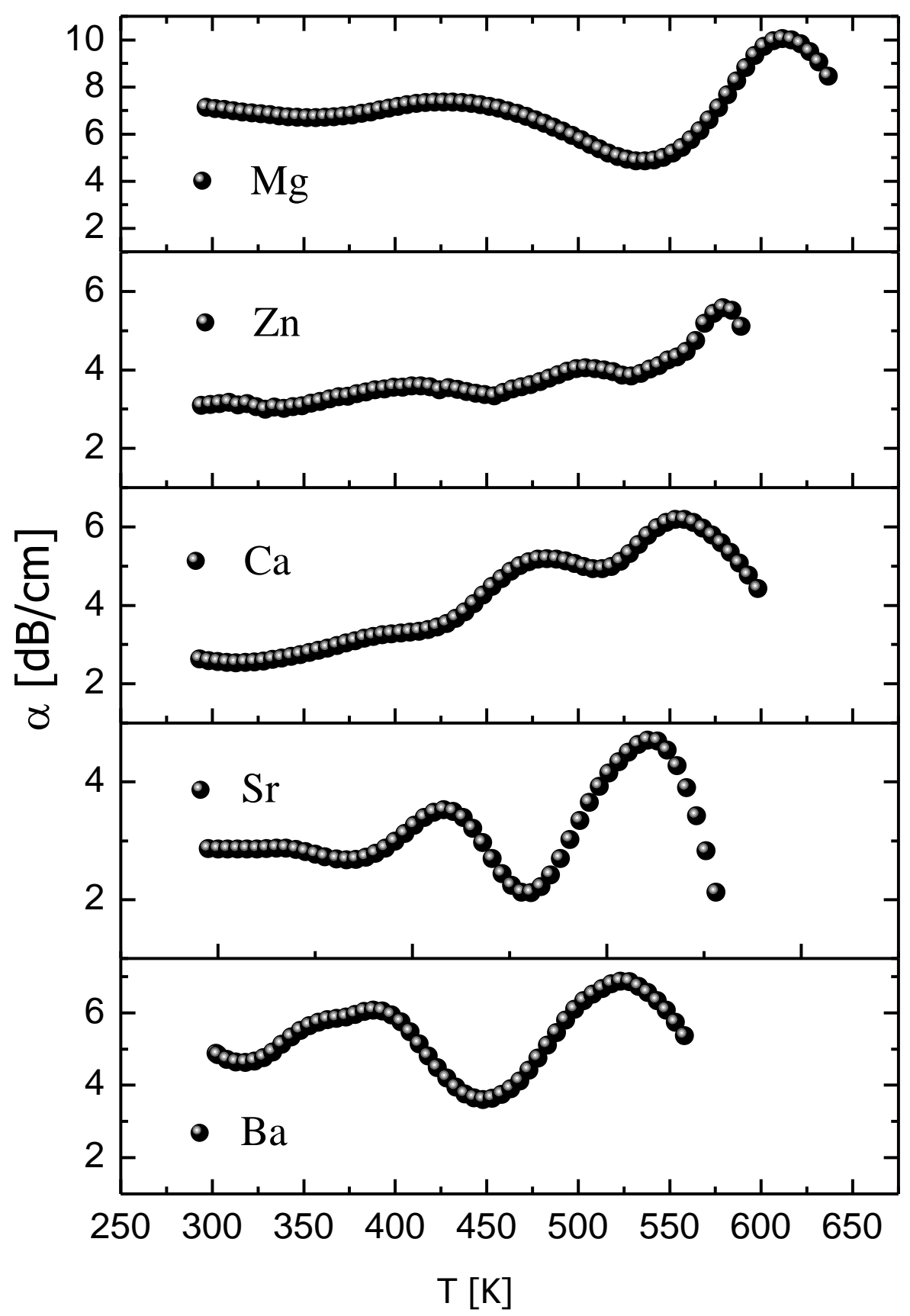

Figure 1 
a)

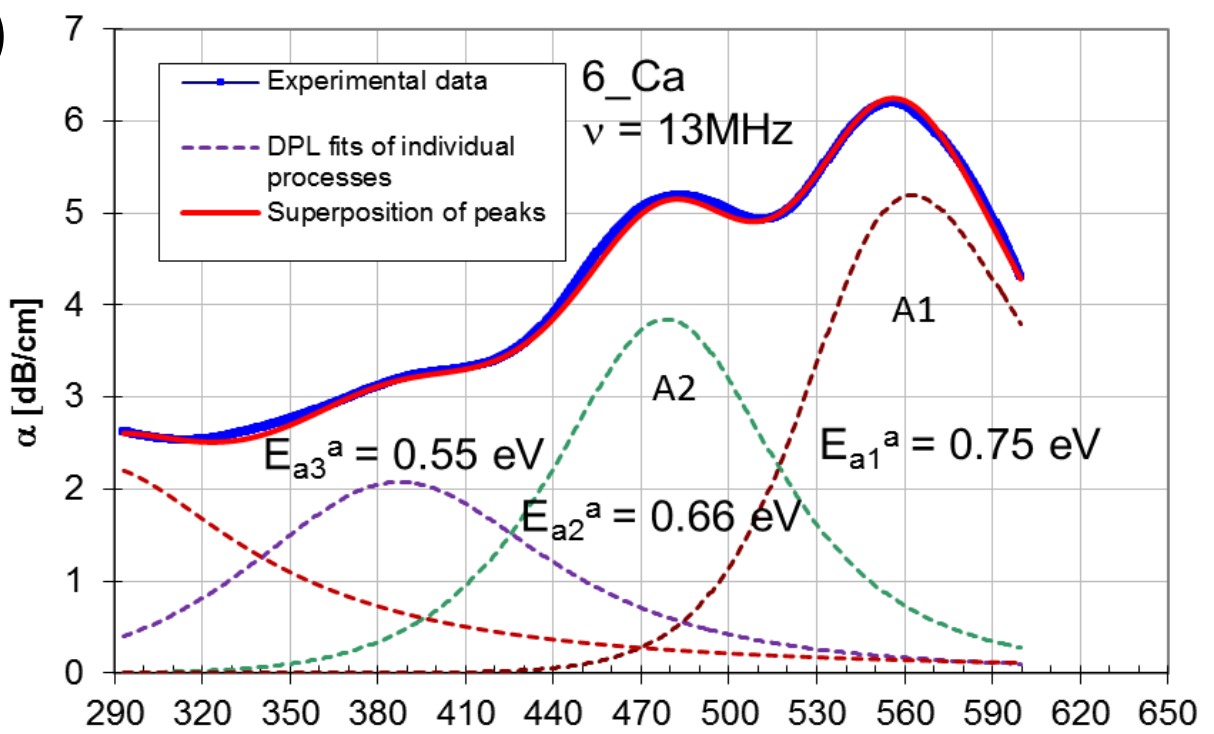

b)

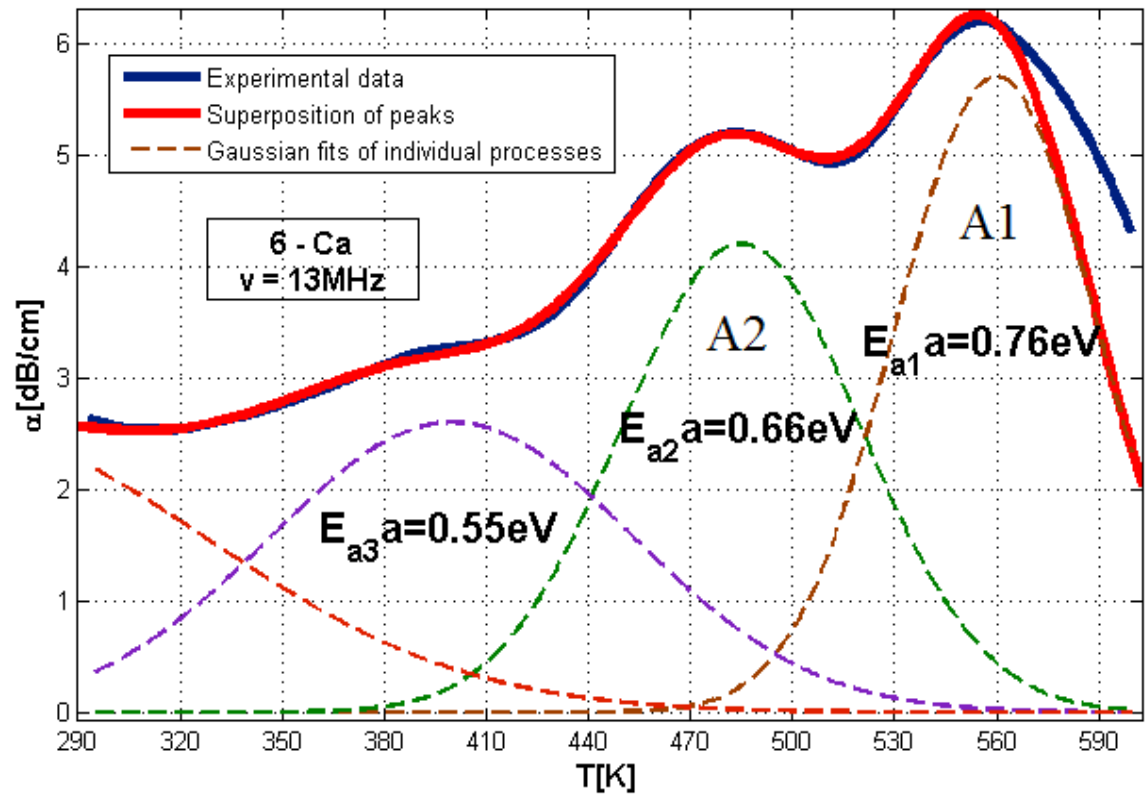

Figure 2 


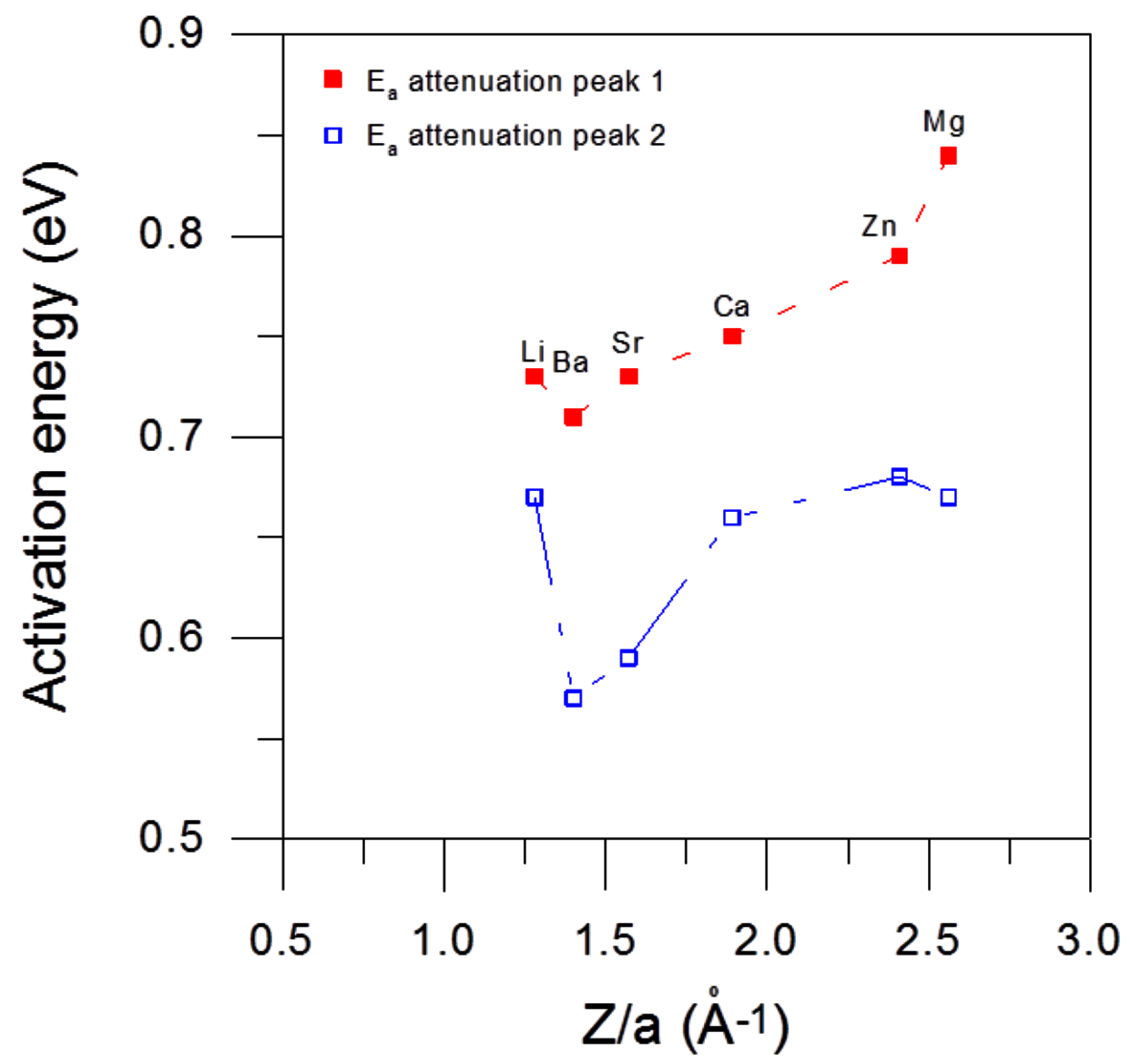

Figure 3 


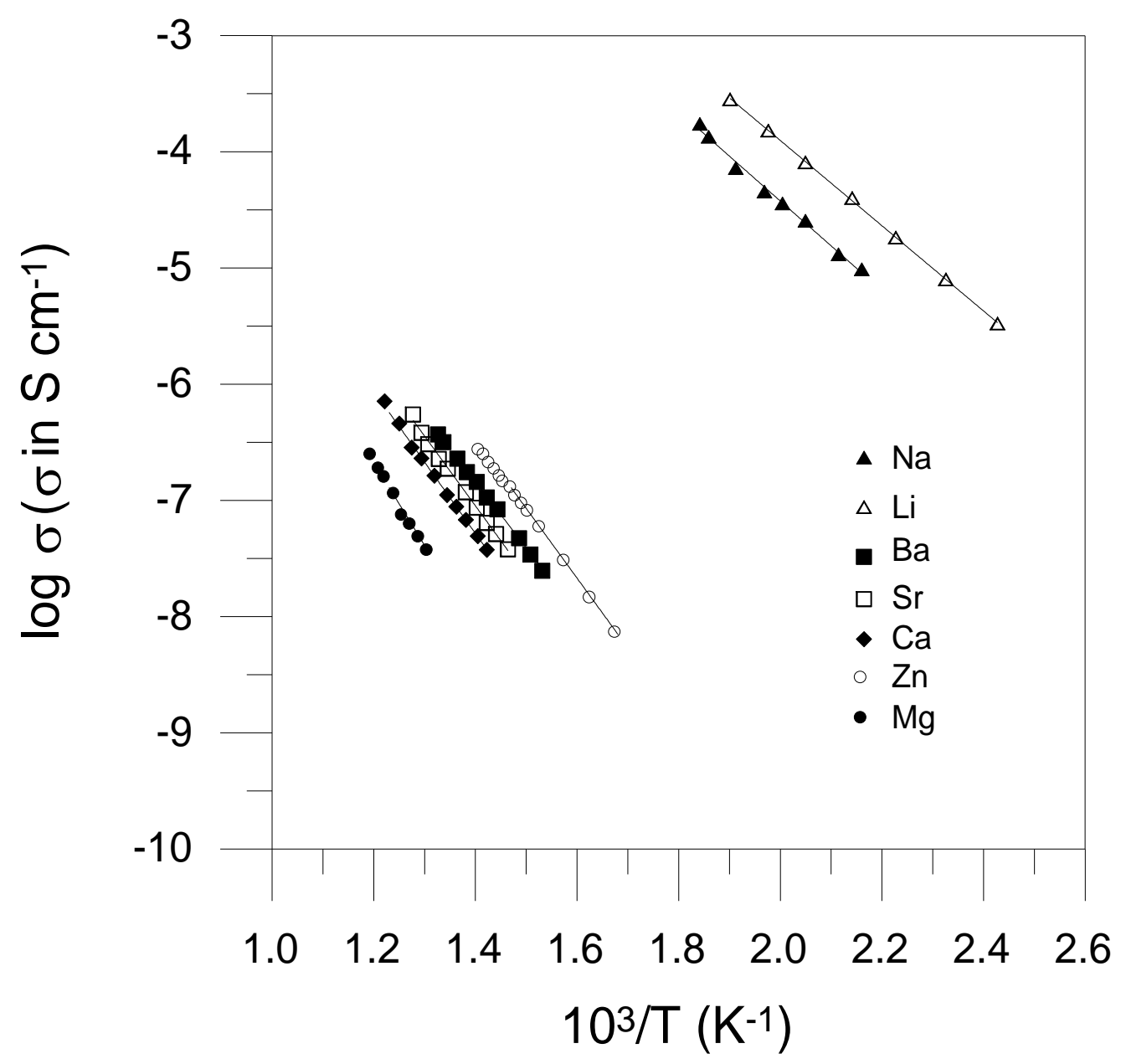

Figure 4 


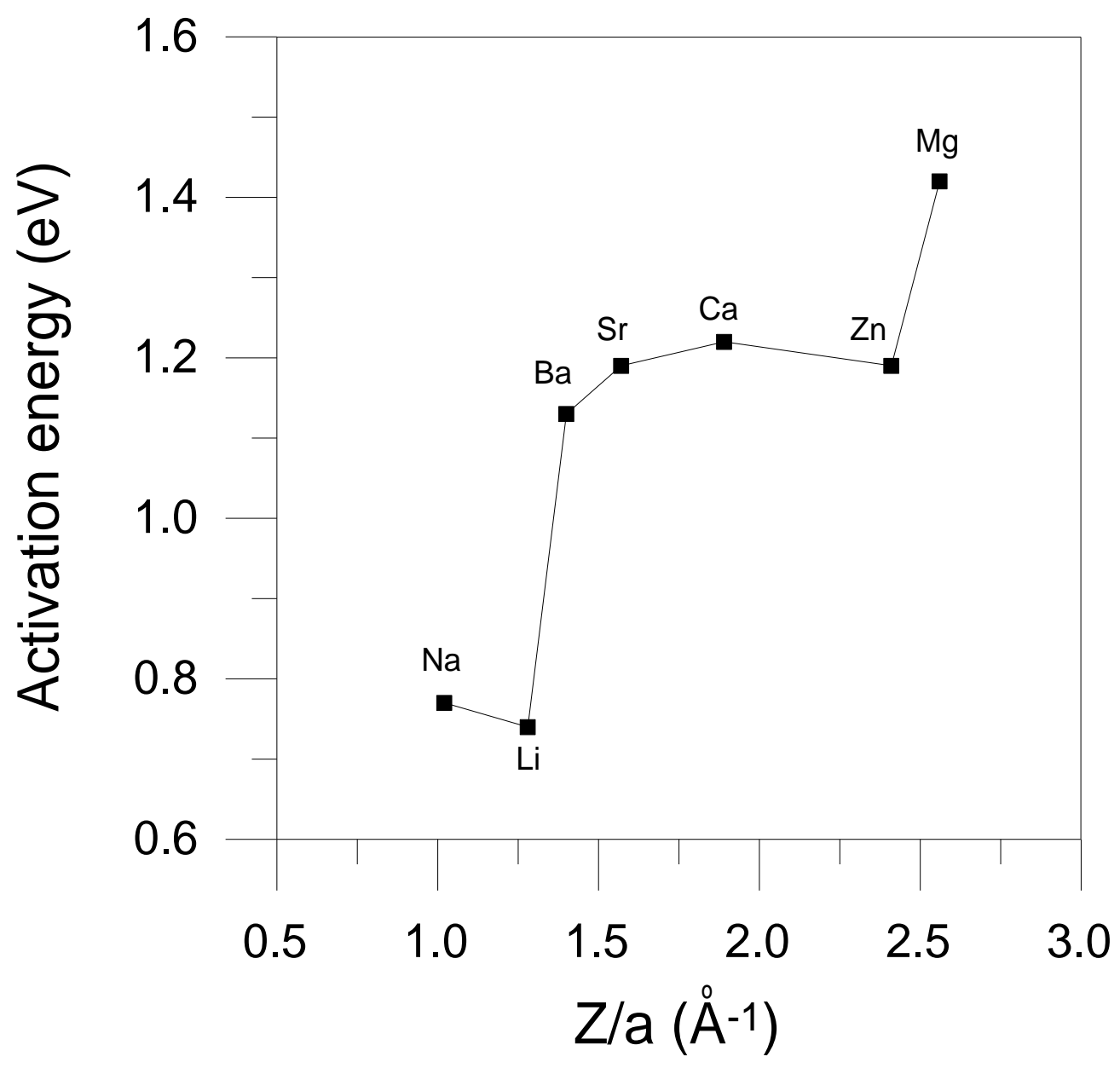

Figure 5 


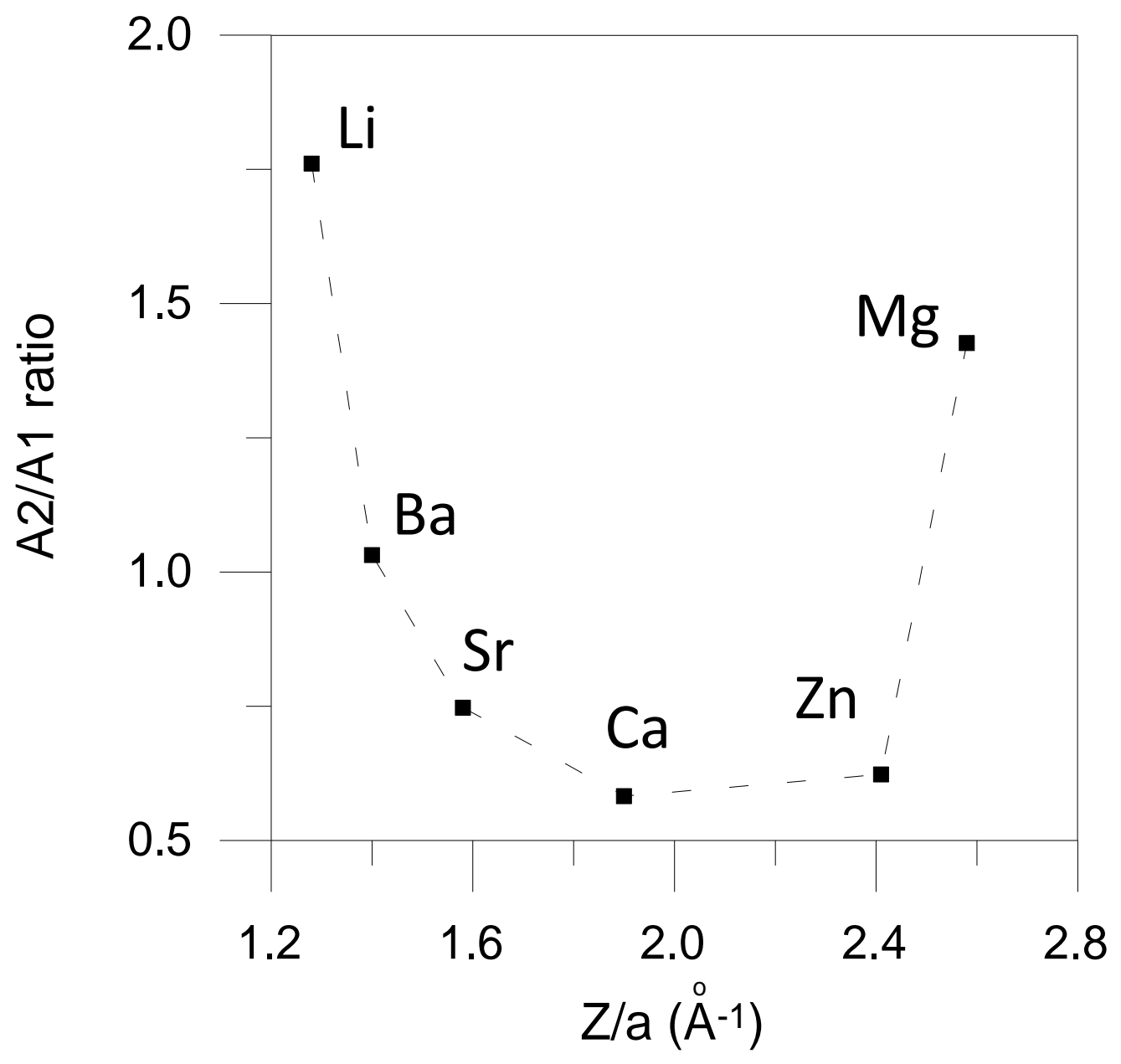

Figure 6 